\title{
Wirkungslogik als Kommunikationsinstrument an den Schnittstellen zwischen Bildungsverwaltung, Bildungswissenschaft und Bildungspraxis
}

\section{Zusammenfassung}

Voraussetzung für eine erfolgreiche Kooperation zwischen Bildungswissenschaft, -verwaltung und -praxis ist ein gemeinsam geteiltes Verständnis der zu bearbeitenden Herausforderungen, Ziele und Lösungsansätze. Im Beitrag wird das Konzept der wirkungsorientierten Steuerung als Kommunikationsinstrument vorgestellt und am Beispiel der sozialräumlichen Vernetzung in Bildungslandschaften eine Wirkungslogik skizziert. Der Einblick in eine qualitative Fallstudie zeigt, wie sich Wissenschaft und Praxis mithilfe dieses Instruments ergänzen können. Abschließend werden Limitationen des Ansatzes aufgezeigt.

Schlüsselwörter: Wirkungslogik, Bildungslandschaft, Schnittstellen

\section{Theory of Change as a Means of Communication between Administration, Science and Practice in Education}

\begin{abstract}
A shared understanding is an important precondition for cooperation between educational science, administration and practice. This article argues that a theory of change approach which is part of impact-oriented planning could be a useful tool for communication. The author proposes a theory of change for cooperation in area-based education networks and gives insights into how science and practitioners can complement each other using this tool and approach. While the approach seems promising, some limitations are also discussed.
\end{abstract}

Keywords: theory of change, education networks, interface management 


\section{$1 \quad$ Einleitung}

In einem idealtypischen Verständnis sind die Schnittstellen zwischen Bildungswissenschaft, Bildungsverwaltung und Bildungspraxis aufeinander abgestimmt: Aus der Bildungspraxis ergeben sich Fragestellungen, die von der Wissenschaft systematisch bearbeitet werden. Daraus ergibt sich Steuerungswissen für die Bildungsverwaltung, welches wiederum als Grundlage für evidenzbasierte Entscheidungen verwendet wird, die die Rahmenbedingungen für die Bildungspraxis abstecken. Allerdings funktioniert der Wissenschafts-Praxis-Transfer im Alltag zumeist nicht nach diesem Muster.

Pädagogische Fachkräfte, Bildungsverwaltung, Bildungspolitik und Bildungswissenschaft haben aufgrund ihrer vielfältigen und komplexen Aufgaben ganz unterschiedliche professionelle Handlungslogiken, Interessen und Kommunikationswege. Darüber hinaus treffen an den Schnittstellen unterschiedliche Professionen und Hierarchieebenen aufeinander. In den Bereichen, wo diese unterschiedlichen Akteure miteinander agieren (sollen), fehlt es an gemeinsamen Verständigungsprozessen, wodurch Kommunikationsprobleme zwischen Steuerungsebene, Praxisakteuren und der Wissenschaft entstehen können. Dies beginnt bereits bei der Verständigung über die Ziele von Bildung. Laut Böttcher (2016) müsste möglichst genau definiert werden, „was im Erziehungs-, Bildungs- oder Qualifizierungsprozess mittels absichtsvoller, methodisch angelegter, organisierter und professionell ausgeführter pädagogischer Interventionen erreicht werden soll“ (Böttcher, 2016, S. 60). Genau diese Verständigung findet aber im Bildungssystem nicht statt - was neben der Steuerung auch die Überprüfbarkeit von pädagogischen Interventionen durch die Wissenschaft erschwert.

Ein gemeinsam getragenes Verständnis der zu bearbeitenden Herausforderungen, Ziele und Lösungsansätze ist allerdings Voraussetzung für erfolgreiche Kooperation an den Schnittstellen zwischen den Systemen. Es gilt daher, mithilfe geeigneter Koordinierungsprozesse darauf hinzuarbeiten, ein gemeinsames Verständnis über die pädagogischen Ziele zu schaffen - und darüber hinaus über die Herausforderungen und möglichen Interventionen auf dem Weg zur Zielerreichung. Dies ist eine anspruchsvolle Aufgabe, da unterschiedliche Handlungslogiken der Akteure unter einem gemeinsamen Dach verortet werden müssen.

Auf kommunaler Ebene kann ein solcher Verständigungsprozess beispielsweise in lokalen Bildungslandschaften durch das kommunale Bildungsmanagement initiiert und begleitet werden. Diesem obliegt es, die gemeinsame Problembearbeitung verschiedener Akteure über Organisationsgrenzen, Zuständigkeitsbereiche, Interessen und Sichtweisen hinweg zu koordinieren (Arbeitsgruppe „Lernen vor Ort“, 2016). In Nordrhein-Westfalen wird diese Funktion insbesondere durch die Regionalen Bildungsbüros übernommen (Manitius \& Berkemeyer, 2011). Diese stellen innerhalb 
der Bildungsverwaltung neben der Steuerungsebene (Landes- und Kommunalpolitik, Schulaufsicht, Schulträger) eine zusätzliche Koordinierungs- und Unterstützungsebene dar und sollen alle vor Ort angestrebten Vernetzungs- und Qualitätssicherungsprozesse koordinieren. Durch Bildungsbüros initiierte Netzwerkstrukturen können zwar eine gute Plattform bieten; für eine tatsächliche Verständigung braucht es aber systematische Kommunikationsprozesse.

Der Ansatz der Wirkungsorientierung bietet Instrumente, um einen Kommunikationsprozess systematisch anzuregen und ein gemeinsames Verständnis zu entwickeln über die Herausforderungen, die zu erreichenden Ziele, die Wirkungsannahmen und Interventionswege und schließlich über die Methoden zur Wirkungsmessung (Klawe, 2006). Dieser Ansatz wird im Folgenden genauer vorgestellt und dann anhand einer prototypischen Wirkungslogik zur sozialräumlichen Vernetzung in Bildungslandschaften angewendet. Dabei wird besonders auf die Rolle der unterschiedlichen Systeme und die idealtypische Zusammenarbeit an den Schnittstellen auf Basis einer gemeinsamen Wirkungslogik eingegangen.

\section{Wirkungsorientierte Steuerung}

Die wirkungsorientierte Steuerung wird im öffentlichen Sektor seit einigen Jahren diskutiert (vgl. z. B. Schröder, 2001). Dieser Steuerungsansatz basiert auf bereits seit den 1950er-Jahren entwickelten Konzepten der „theory-driven evaluation“ (für eine ausführliche Übersicht vgl. Funnell \& Rogers, 2011). Bei eher klassischen Evaluationsund Steuerungsansätzen stehen die (häufig quantitative) Messung der Zielerreichung oder die konkreten Auswirkungen der Maßnahme auf Nutzerinnen im Mittelpunkt. Im Gegensatz dazu zielen wirkungsorientierte Ansätze darauf, die Wirkung von komplexen sozialen Programmen zu messen, indem Zusammenhänge zwischen Aktivitäten, Ergebnissen und den Kontextbedingungen einer sozialen Initiative systematisch und kumulativ erforscht werden (Connell \& Kubisch, 1998). Grundlage dafür ist eine Wirkungslogik oder Programmtheorie, die aufzeigen soll „how the program works, with whom, and under what circumstances" (Astbury \& Leeuw, 2010, S. 365). In Bezug auf Bildungslandschaften und das kommunale Bildungsmanagement sind Vorschläge für eine Übertragung entwickelt worden (Jungermann, 2018; Niedlich, Lindner \& Brüsemeister, 2014).

Die wirkungsorientierte Steuerung basiert auf drei Elementen: den Wirkungszielen, der Wirkungslogik und dem Wirkungsmonitoring (vgl. Abb. 1 auf der folgenden Seite und im Folgenden Schröder \& Kettiger, 2001; Jungermann, 2018). 
Abb. 1: Vorgehen bei der wirkungsorientierten Steuerung (Jungermann, 2018)

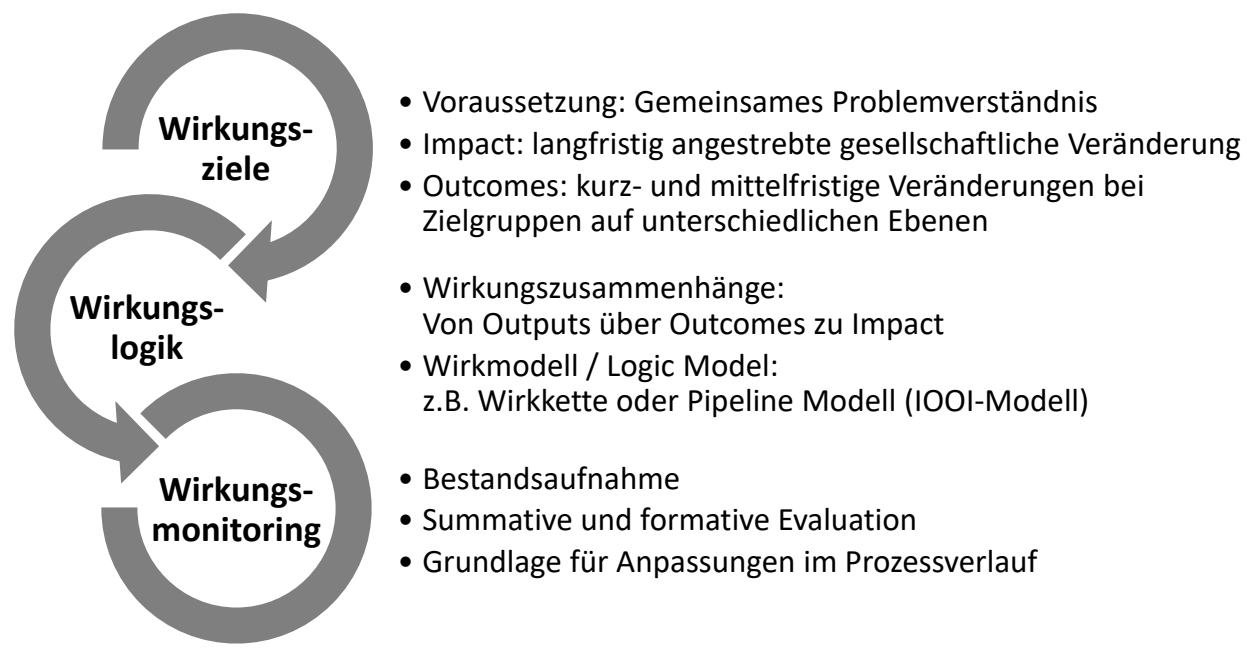

Quelle: eigene Darstellung

Wirkungsziele: Voraussetzung ist, dass die Beteiligten ein gemeinsames Problemverständnis bezüglich der Bedürfnisse der Zielgruppen haben und dieses auch expliziert wird. Auf dieser Basis erfolgt eine Einigung über die $\mathrm{zu}$ erreichenden Wirkungsziele, also über die langfristigen Ziele für gesellschaftliche Veränderungen (Impacts) (Funnell \& Rogers, 2011). Neben den langfristigen Zielen werden auch mittel- und kurzfristige Ziele (Outcomes) für unterschiedliche Zielgruppen festgelegt, die ihrerseits Voraussetzung sind, damit die Wirkungsziele erreicht werden können.

Wirkungslogik: Die eigentliche Wirkungslogik (auch Programmtheorie oder Theory of Change genannt) legt dar, welche Leistungen und Maßnahmen (Outputs) nötig sind, um die angestrebten Ziele $\mathrm{zu}$ erreichen. Dabei werden Wirkungszusammenhänge zwischen den langfristigen Zielen (Impact), kurz- und mittelfristigen Ergebnissen (Outcomes) und den dafür geplanten Maßnahmen und Aktivitäten (Outputs) hergestellt und die dafür benötigten Ressourcen, Rahmenbedingungen und Kontextfaktoren (Inputs) präzisiert (Funnell \& Rogers, 2011; Donaldson, 2007). Die Wirkungslogik kann zwar auf Erfahrungswissen der Akteure basieren, sollte aber idealerweise auch durch anerkannte wissenschaftliche Theorien unterfüttert werden. Durch die Erstellung einer Wirkungslogik werden die dem Programm zugrundeliegenden Wirkannahmen explizit gemacht und zu einer schlüssigen Wirkungskette zusammengefügt (Donaldson, 2007). Die gemeinsame Erarbeitung der Wirkungslogik zwischen den Beteiligten dient dabei als wichtiges Kommunikationsmoment, um eine gemeinsame Verständigung zu erzielen. 
Wirkungsmonitoring: Im Rahmen des Wirkungsmonitorings werden die Plausibilität der Wirkungslogik überprüft und die einzelnen Schritte gemessen. Das Monitoring nimmt dabei den gesamten Prozess der Planung und Umsetzung in den Blick. Dabei wird insbesondere dargestellt, wie schlüssig die einzelnen Schritte der Wirkungslogik aufeinander abgestimmt sind, wo konzeptionelle Lücken vorliegen und ob relevante Zwischenschritte im Prozess erreicht wurden (Donaldson, 2007). Dafür können ganz unterschiedliche - sowohl qualitative als auch quantitative - Datenquellen genutzt werden.

Insgesamt handelt es sich beim wirkungsorientierten Vorgehen nicht um einen unumstößlich festgelegten Rahmen. Die Wirkungslogik kann in einem iterativen und adaptiven Prozess auf Basis des Wirkungsmonitorings regelmäßig überprüft, überarbeitet und an veränderte Rahmenbedingungen und Erfahrungswerte im Prozessverlauf angepasst werden (Funnell \& Rogers, 2011).

\subsection{Wirkungsorientiertes Vorgehen als Kommunikationsinstrument für Verständigungsprozesse}

Kerninstrument für die Wirkungsorientierung ist die Erarbeitung einer Wirkungslogik. Sie ist leitend für die Evaluation von Initiativen und ein wichtiges Steuerungs-, Planungs- und Managementinstrument. Besonders in komplexen sozialen Programmen, in denen unterschiedliche Akteure, Interessen und Handlungslogiken auf komplexe soziale Herausforderungen treffen, bietet die Wirkungslogik einen Handlungsrahmen für die Koordination von Aktivitäten und dient dazu, verschiedene Akteure mit unterschiedlichen Interessen zu einem gemeinsamen und koordinierten Handeln zu bewegen (Donaldson, 2007; Klawe, 2006). Daher sollte sie idealerweise bereits zu Beginn der Konzeptionsphase von Maßnahmen und Initiativen entwickelt werden und handlungsleitend für die gesamte Programmdurchführung sein, auch wenn sie im Prozess bei Bedarf Modifikationen erfährt (Donaldson, 2007; Connell \& Kubisch, 1998).

Die gemeinsame, dialogische Erarbeitung einer Wirkungslogik und Verständigung über Ziele, Interventionsschritte und Wirkannahmen hilft im Kommunikations- und Klärungsprozess zwischen verschiedenen Akteuren auf unterschiedlichen Ebenen (Chen, 1990; Patton 1986). Akteure bringen abhängig von ihrer professionellen Erfahrung oder ihrem wissenschaftlichen Hintergrund unterschiedliche implizite oder explizite, manchmal auch konfligierende Annahmen mit (Klawe, 2006). Bei der Aushandlung einer Wirkungslogik werden diese transparent gemacht und in einem gemeinsamen Verständigungsprozess zusammengefügt (Donaldson, 2007; Klawe, 2006). Eine gute Wirkungslogik zeichnet sich dadurch aus, dass diese von allen als plausibel, umsetzbar und verifizierbar angesehen wird (Connell \& Kubisch, 1998). 
Ziel des Aushandlungs- und Verständigungsprozesses ist es, dass die Intervention bei allen beteiligten Akteuren legitimiert wird. Damit soll auch gesichert werden, dass die involvierten Personen bei der Implementation, aber auch dem damit zusammenhängenden Wirkungsmonitoring mitwirken (Donaldson, 2007).

\section{Wirkungsorientierung bei der sozialräumlichen Vernetzung in Bildungslandschaften}

Im Folgenden soll verdeutlicht werden, wie ein wirkungsorientiertes Vorgehen dazu beitragen kann, an der Schnittstelle zwischen Forschung, pädagogischer Praxis und Steuerungsebene zu vermitteln. Dabei wird das Beispiel der sozialräumlichen Vernetzung von Schule im Sinne einer Bildungslandschaft genauer betrachtet.

Sozialraumvernetzung zwischen den unterschiedlichen Akteuren ist ein zentrales Element des Diskurses zu Bildungslandschaften. Es fehlt bisher allerdings an einer klaren theoretischen Rahmung, die erklärt, wie die Zusammenarbeit zwischen Akteuren im Sozialraum zu einer ganzheitlichen Persönlichkeitsentwicklung der Kinder und Jugendlichen und damit zu mehr Bildungsgerechtigkeit beitragen kann. Dies soll anhand einer (vereinfachten, prototypischen) Wirkungslogik hier aufgezeigt werden (für eine ausführliche Darstellung vgl. Jungermann, 2018).

Die Überlegungen zu dem hier vorgeschlagenen wirkungsorientierten Vorgehen basieren auf den theoretischen Annahmen des sozioökologischen Modells des amerikanischen Entwicklungspsychologen Bronfenbrenner (1979). Auf dieser Basis wurden Wirkungsziele und eine Wirkungslogik abgeleitet (vgl. ausführlich Jungermann, 2018). Die Wirkungslogik ist als prototypischer Vorschlag zu verstehen, der von den Akteuren auf die lokalen Gegebenheiten angepasst werden kann.

\subsection{Wirkungsziele in Bildungslandschaften}

Zunächst gilt es, sich über die zu erreichenden Wirkungsziele (Impact und Outcomes auf verschiedenen Ebenen) zu verständigen, die mit der sozialräumlichen Vernetzung erreicht werden sollen.

\section{Impact}

Allgemeines Ziel von Bildungslandschaftsinitiativen ist es, Chancengerechtigkeit zu erhöhen. Im Sinne eines in Bildungslandschaften verfolgten erweiterten Bildungsverständnisses könnte ein Impact-Ziel also wie folgt formuliert werden:

Alle Kinder und Jugendlichen der Bildungslandschaft erhalten unabhängig von ihrer familiären Herkunft die Kompetenzen für ein gelingendes Leben und die 
gleichberechtigte Teilhabe an der Gesellschaft. Dies umfasst eine ganzheitliche Persönlichkeitsentwicklung, also nicht nur die primär in der Schule vermittelten fachlich-kognitiven, sondern auch psycho-emotionale und soziale Kompetenzen, Haltungen und Werte.

\section{Outcomes}

Verkürzt dargestellt, geht Bronfenbrenner (1979) davon aus, dass die individuelle Entwicklung in einem sozialen Kontext stattfindet, der mehrere Ebenen umfasst. Diese sind die Individualebene, das Mikrosystem (direkte Beziehungen zwischen dem Individuum und Personen im Umfeld), das Mesosystem (Beziehungen zwischen verschiedenen Akteuren des Mikrosystems), das Exosystem (Beziehungen zwischen Mikrosystem und weiteren Akteuren, mit denen das Individuum nicht im direkten Kontakt steht) sowie das Makrosystem (gesellschaftlicher Kontext). Bildungslandschaften betreffen insbesondere das Mikro-, Meso- und Exosystem, weshalb für alle drei Ebenen Outcome-Ziele formuliert werden:

Auf Mikro-Ebene findet Persönlichkeitsentwicklung durch proximale Prozesse statt (Bronfenbrenner, 1979). Proximale Prozesse sind reziproke Interaktionen eines Individuums mit Bezugspersonen (aber auch Objekten und Symbolen) im direkten Umfeld. Um zur Persönlichkeitsentwicklung beizutragen, sollten proximale Prozesse vielfältig, anregend und qualitativ hochwertig sein, regelmäßig über einen längeren Zeitraum erfolgen und in ihrer Komplexität dem Entwicklungsstand des Individuums angepasst sein.

Ein Outcome-Ziel der sozialräumlichen Vernetzung in einer Bildungslandschaft für das Mikro-System, also die Beziehungen zwischen den Adressaten und den pädagogischen Fachkräften, könnte entsprechend wie folgt formuliert werden:

Outcome Mikro-System (pädagogische Beziehungen)

Alle Kinder und Jugendlichen erleben vielfältige, anregende proximale Prozesse in formalen und non-formalen pädagogischen Settings der Bildungslandschaft.

Voraussetzung dafür ist, dass einerseits ein möglichst reichhaltiges ganzheitliches Bildungsangebot entlang der Bildungsbiographie der Kinder und Jugendlichen existiert und dass dies auch von benachteiligten Zielgruppen genutzt wird, also keine Selektionseffekte entstehen. Andererseits erfordert dies eine Haltung der pädagogischen Fachkräfte, die ganzheitliche Persönlichkeitsentwicklung und Potenzialorientierung ins Zentrum ihrer Arbeit stellt. 
Im Meso-System werden nach Bronfenbrenner (1979) proximale Prozesse zudem begünstigt, wenn die beteiligten Akteure (z.B. Schulen, außerschulische Partner, Jugendhilfe) im Meso-System unterstützende Verbindungen schaffen. Diese werden von Bronfenbrenner nicht präzise definiert. Auf Basis von Bronfenbrenners Erläuterungen wurden im Rahmen einer qualitativen Fallstudie Indikatoren und darauf aufbauend ein Analyseraster für unterstützende Verbindungen entwickelt (Jungermann, eingereicht). Diese zeichnen sich demnach durch Reziprozität, komplexe Kooperationsaktivitäten und Institutionalisierung aus (ebd.). Reziprozität bedeutet, dass Akteure sich gegenseitig kennen, einander auf Augenhöhe begegnen und den Beitrag der unterschiedlichen Professionen zum gemeinsamen Ziel gegenseitig wertschätzen. In komplexen Kooperationsbeziehungen werden über den punktuellen Austausch hinaus im Sinne der Ko-Konstruktion gemeinsame Inhalte entwickelt. Institutionalisiert sind Beziehungen, wenn sie systematisch organisational verankert sind und nicht allein auf dem Engagement einzelner Personen beruhen.

Outcome Meso-System (kooperierende Einrichtungen)

Die kooperierenden Einrichtungen im Umfeld der Kinder und Jugendlichen gehen unterstützende Verbindungen ein, also reziproke, komplexe und institutionalisierte Kooperationsbeziehungen.

Im Exosystem entstehen die nötigen Rahmenbedingungen auf Ebene der kooperierenden Einrichtungen, wenn die übergeordneten Koordinierungs- und Steuerungsebenen (z.B. Schulträger, Schulaufsicht, Verwaltung, Jugendhilfe, aber auch Förderpartner und Wirtschaftsunternehmen) die Vernetzung finanziell, politisch und organisatorisch unterstützen.

Outcome Exo-System (Steuerungs- und Koordinierungsebene)

Die politischen und finanziellen Rahmenbedingungen für die Vernetzung sind geschaffen, die Vernetzung wird unterstützt und koordiniert.

\subsection{Prototypische Wirkungslogik zur Vernetzung in Bildungslandschaften}

Abbildung 2 auf der folgenden Seite zeigt die aus diesem Narrativ abgeleitete Wirkungslogik und die jeweils notwendigen Outputs/Maßnahmen auf den unterschiedlichen Ebenen (Individualebene, Mikro-, Meso- und Exosystem): 
Abb. 2: Wirkungslogik zur sozialräumlichen Vernetzung in Bildungslandschaften

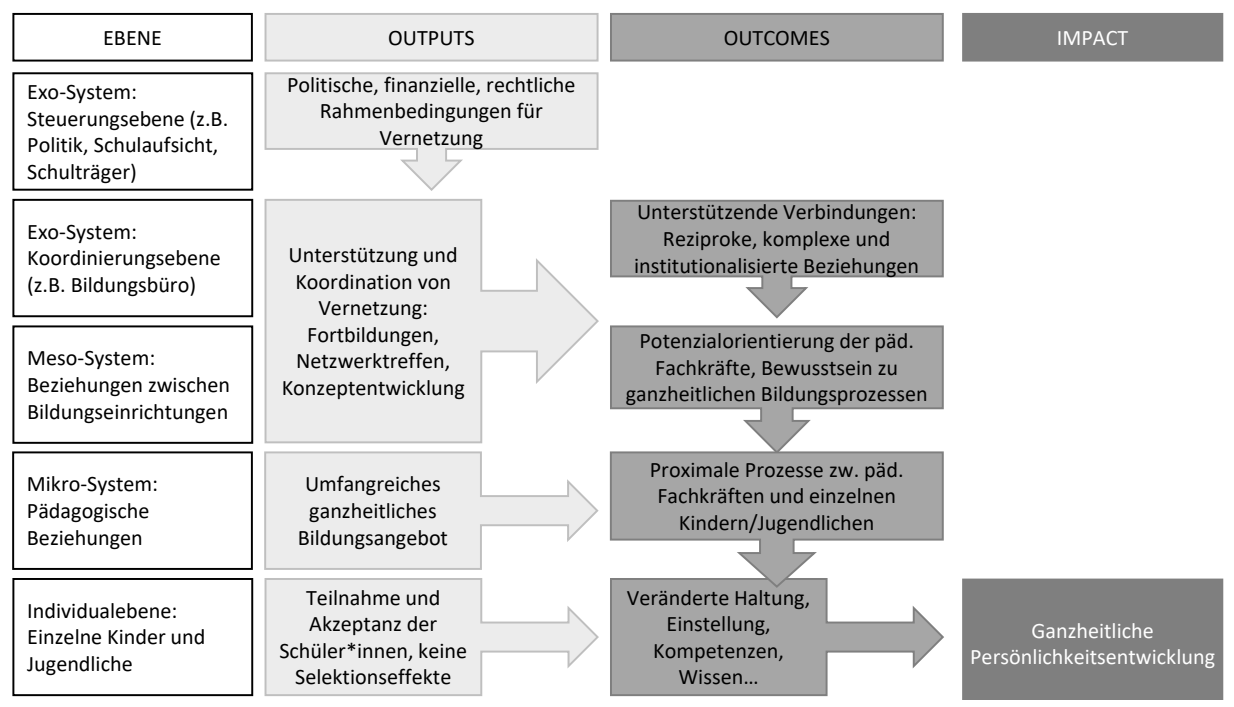

Quelle: eigene Darstellung

Auf Basis dieser konzeptionellen Überlegungen können Konsequenzen für die pädagogische Praxis, das Steuerungs-/Koordinierungshandeln sowie die Wissenschaft abgeleitet werden.

\subsection{Ableitungen für pädagogische Praxis, Koordinierungs- und Steuerungsebene sowie Wissenschaft}

Für die pädagogische Praxis ergibt sich aus dieser Wirkungslogik die Frage, welche konkreten Maßnahmen (Outputs) erforderlich sind, um die verschiedenen Zwischenziele zu erreichen. Aus Schulentwicklungsperspektive müsste beispielsweise zunächst ein Überblick über die vorhandenen und potenziellen Partner erfolgen und eingeschätzt werden, welche Qualität die Kooperationen in Bezug auf Reziprozität, Kooperationsaktivität und Institutionalisierung haben (siehe dazu Einblicke aus einer Fallstudie im folgenden Abschnitt). Zudem könnten konkrete Maßnahmen, wie beispielsweise Fortbildungen und gemeinsame Fachtage, umgesetzt werden, um ein gemeinsames pädagogisches Verständnis des multiprofessionellen schulischen Teams und der pädagogischen Fachkräfte im Sozialraum in Bezug auf ganzheitliche Bildung und Potenzialentfaltung zu entwickeln.

Aus Koordinierungs- und Steuerungsperspektive sollten Kooperationsbeziehungen unterstützt werden. So könnte beispielsweise die Aufgabe der Bildungsbüros sein, entsprechende Netzwerkaktivitäten zu koordinieren oder im Sinne eines Wissens- 
managements den Schulen einen Überblick über potenzielle Partner im Sozialraum zu geben. Die Steuerungsebene wiederum sollte die finanziellen und politischen Rahmenbedingungen schaffen, zum Beispiel Ressourcen zur Begleitung der Netzwerkarbeit oder für Fortbildungen bereitstellen.

Aufgabe der Wissenschaft wäre es, die Plausibilität und damit die Qualität der Wirkungslogik und der ihr zugrundeliegenden Kausalannahmen zu bewerten (Böttcher, 2016). Beispielsweise kann sie durch ergänzende Theorien oder empirische Erkenntnisse unterfüttert werden. Durch eigene Forschungsdesigns können darüber hinaus einzelne Wirkannahmen überprüft werden: Führen die Unterstützungs- und Koordinierungstätigkeiten tatsächlich zu einer verbesserten Kooperation zwischen den Akteuren? Welche Haltung haben pädagogische Fachkräfte unterschiedlicher Professionen in Bezug auf das Impact-Ziel ganzheitliche Bildung? Wie sieht die tatsächliche Kooperation im Sozialraum aus? etc.

Es obliegt der Wissenschaft im Rahmen des Wirkungsmonitorings zudem, die Zielerreichung der verschiedenen Wirkungsziele mit empirisch am Fall operationalisierbaren Indikatoren zu messen. Dazu müssen gegebenenfalls neue qualitative und quantitative Erhebungsinstrumente entwickelt werden. Wie können beispielsweise unterstützende Verbindungen in einem Sozialraum gemessen werden? Wie lassen sich proximale Prozesse nachweisen? Wie kann erhoben werden, dass die Zielgruppen tatsächlich erreicht werden und keine Selektionsprozesse vorliegen?

Insgesamt greifen also auf Basis einer gemeinsam getragenen Wirkungslogik die konkreten pädagogischen Maßnahmen, die Steuerungs- und Koordinierungsebene und die Wissenschaft ineinander. Die Wirkungslogik dient dabei als Kommunikationsinstrument, die in einem iterativen und dialogischen Prozess immer wieder verfeinert, überarbeitet, an neue Gegebenheiten in der Umsetzungspraxis bzw. an neue Erkenntnisse aus der wissenschaftlichen Begleitung angepasst wird. Ziel ist, gemeinsam herauszufinden, was genau unter welchen konkreten Bedingungen für wen funktioniert. Die Rolle der Wissenschaft ist also hier nicht die eines externen, objektiven Außenseiters. Vielmehr wird im Sinne einer "developmental evaluation“ (Patton, 2011) der gesamte Prozess der Datenerhebung und Interpretation gemeinsam und partnerschaftlich mit den handelnden Akteuren durchgeführt, um schnell und zeitnah Erkenntnisse in adaptierte Praxis einfließen zu lassen. Aus dem Dialog ergeben sich somit neue, praxisrelevante Fragestellungen für die Forschung; gleichzeitig liefert die wissenschaftliche Begleitung relevante Informationen für das pädagogische Handeln und für Steuerungsentscheidungen, die im Idealfall direkt aufgegriffen und umgesetzt werden können. 


\section{Einblick aus einer Fallstudie zur sozialräumlichen Vernetzung einer Schule}

Im Folgenden wird mit einer qualitativen Fallstudie beispielhaft skizziert, wie sich Wissenschaft, Koordinierungsebene und pädagogische Praxis ergänzen können (Jungermann, eingereicht). Im Rahmen der Fallstudie wurde ein Analyseinstrument entwickelt, mit dem die unterstützenden Verbindungen im Sozialraum erhoben werden können. Untersucht wurde das Kooperationsnetzwerk einer weiterführenden Schule in herausfordernder Lage im Ruhrgebiet. Anhand von Netzwerkkarten und Experteninterviews mit verschiedenen schulischen Akteuren (Schulleitung, Sozialpädagog^innen, Lehrkräfte) wurde aufgezeigt, dass die Schule eine Vielzahl von Kooperationsaktivitäten eingeht, sie allerdings kein systematisches Vorgehen verfolgt, um die Kooperationen bestmöglich - im Sinne der oben skizzierten Wirkungslogik zur Förderung der individuellen Persönlichkeitsentwicklung der Schülerinnen zu gestalten.

Zunächst wurden fünf Kooperationsbereiche identifiziert, denen die Partner und die pädagogischen Ziele zugeordnet werden können: Einzelfallunterstützung, außerunterrichtliche Aktivitäten, Berufsorientierung, Schul-/Unterrichtsentwicklung sowie Sozialraumentwicklung. Um die einzelnen Kooperationsbereiche genauer zu betrachten, wurde angelehnt an die (eher vagen) Ausführungen Bronfenbrenners (1979) zu unterstützenden Verbindungen ein Analyseraster auf Basis des Interviewmaterials entwickelt. Für die drei im vorherigen Abschnitt erklärten Analysedimensionen Reziprozität, Komplexität der Kooperationsaktivität und Institutionalisierung (vgl. Kap. 3.1) wurden dazu Indikatoren aus dem Material identifiziert und die Kooperationsbereiche auf einer Skala eingeordnet. Dabei zeigte sich im Rahmen der Fallstudie, dass die Kooperationsbereiche von der Schule unterschiedlich systematisch bearbeitet werden (vgl. Abb. 3 auf der folgenden Seite).

So sind beispielsweise Kooperationen im Bereich der Einzelfallunterstützung und der Berufsorientierung an dieser Schule sehr weit ausgeprägt. Viele Kooperationspartner sind darüber hinaus im Bereich der außerunterrichtlichen Aktivitäten angesiedelt; dieser wird von vielen interviewten Lehrkräften als bedeutsam für die Persönlichkeitsentwicklung und auch für die Schüler*innen-Lehrkräfte-Beziehung angesehen. Allerdings sind Kooperationen in diesem Bereich weit weniger institutionalisiert, weniger komplex und basieren häufig auch nicht auf reziproken Beziehungen. Das bedeutet unter anderem, dass die Relevanz dieser Beziehungen nicht vom gesamten Kollegium als gleichermaßen wichtig angesehen wird. Die Kooperation mit weiteren Einrichtungen im Sozialraum ist dagegen nur sehr gering bis gar nicht ausgeprägt. Insgesamt gibt es an der Schule kein klares Konzept darüber, mit welchen konkreten Zielsetzungen Kooperationen eingegangen werden und wie diese systematisch gestaltet werden können. 
Wirkungslogik als Kommunikationsinstrument |

Abb. 3: Systematischer Vergleich der Kooperationsbereiche nach Analysedimensionen (vgl. Jungermann, eingereicht; eigene Übersetzung aus dem Englischen)

\begin{tabular}{|c|c|c|c|}
\hline Reziprozität & Niedrig & Mittel & Hoch \\
\hline Kooperationsaktivität & Austausch & Arbeitsteil. Kooperation & Ko-Konstruktion \\
\hline Institutionalisierung & Niedrig & Mittel & Hoch \\
\hline \multirow{3}{*}{ Einzelfallunterstützung } & & & $x$ \\
\hline & & & $\mathbf{x}$ \\
\hline & & & $\mathbf{x}$ \\
\hline \multirow{3}{*}{$\begin{array}{l}\text { Außerunterrichtliche } \\
\text { Aktivitäten }\end{array}$} & $\mathbf{x}$ & (x) & \\
\hline & & $\mathbf{x}$ & \\
\hline & $x$ & & \\
\hline \multirow{3}{*}{ Berufsorientierung } & & $\mathbf{x}$ & \\
\hline & & $\mathbf{x}$ & (x) \\
\hline & & & $\mathrm{x}$ \\
\hline \multirow{3}{*}{$\begin{array}{l}\text { Schul-/Unterrichts- } \\
\text { entwicklung }\end{array}$} & $\mathrm{x}$ & & \\
\hline & $x$ & & (x) \\
\hline & $x$ & & \\
\hline \multirow{3}{*}{ Sozialraumentwicklung } & $\mathrm{x}$ & & \\
\hline & $\mathbf{x}$ & & \\
\hline & $\mathbf{x}$ & & \\
\hline
\end{tabular}

$x$ Allgemeine Bewertung über alle Kooperationsverbindungen im Kooperationsbereich

(x) Einzelne Abweichungen innerhalb des Kooperationsbereichs

Quelle: eigene Darstellung

Ziel der Fallstudie war es zunächst, das Kooperationsnetz der Schule zu erheben. Anschließend daran könnte man nun die oben skizzierte Wirkungslogik für Bildungslandschaften als Kommunikationsinstrument nutzen, um im Dialog zwischen Wissenschaft, Koordinierungsebene und Praxis konkrete Maßnahmen für die Stärkung der Kooperation im Sozialraum abzuleiten. So können beispielsweise Zielsetzungen für die einzelnen Kooperationsbereiche formuliert werden. Es kann aufgezeigt werden, wo Lücken sind und welche Kooperationsbereiche qualitativ (in Bezug auf Reziprozität, Kooperationsaktivität und Institutionalisierung) noch ausgebaut werden sollten.

Für die Koordinierungsebene lässt sich ableiten, welche Maßnahmen ergriffen werden sollten, um die Kooperationen in bestimmten Bereichen zu unterstützen. 
Die Aufgabe der Wissenschaft wäre dann, die Annahmen, die den auf Praxis- und Koordinierungsebene ergriffenen Maßnahmen zugrunde liegen, zu plausibilisieren und deren Wirkungen im Blick zu halten. Das hier vorgestellte Analyseraster kann dabei als leicht anzuwendendes Selbsteinschätzungsinstrument im Rahmen des Wirkungsmonitorings verwendet werden.

\section{$5 \quad$ Fazit und Limitationen der Wirkungsorientierung}

Die wirkungsorientierte Steuerung bietet einen vielversprechenden Ansatz, um Wissenstransfer zwischen Wissenschaft, Bildungsverwaltung und schulischer Praxis zu ermöglichen. Das dialogische und kooperative Vorgehen bei der Erstellung der Wirkungslogik, beim Monitoring und bei der Strategieanpassung eröffnet die Möglichkeit, durch Kommunikations- und Aushandlungsprozesse zu einem gemeinsamen Situationsverständnis, einem abgestimmten Vorgehen und $\mathrm{zu}$ synergetischem Handeln im Sinne der Zielerreichung zu führen. Anstelle eines linear vollzogenen Wissenstransfers (Erkenntnisse der Wissenschaft > Steuerungsentscheidung der Bildungsverwaltung > pädagogische Umsetzung an den Schulen), der sich in dieser Form als nicht realistisch gezeigt hat, tritt ein kooperativ zu gestaltender Kommunikationsprozess. Dabei werden die unterschiedlichen Perspektiven (pädagogische Fachkräfte, Steuerungsakteure und Wissenschaft) zusammengeführt und können voneinander profitieren. Im Idealfall können so Forschungsdesigns in einem gemeinsamen Prozess entwickelt werden, was einerseits die Praxisrelevanz der wissenschaftlichen Studien erhöht und andererseits im Idealfall für eine größere Akzeptanz der Forschungsergebnisse und damit für besseren Wissenstransfer sorgt. Die Akteure der Bildungsverwaltung - insbesondere die Regionalen Bildungsbüros - können hier als koordinierende Instanzen auftreten, indem sie beispielsweise beim Wissenstransfer und der Erarbeitung von Wirkungslogiken vermitteln.

Nachfolgend soll auf einige Limitationen der Wirkungsorientierung hingewiesen werden. Grundsätzlich können bei der Entwicklung einer Wirkungslogik Probleme auftreten, die bereits Weiss zusammenfasst (für den folgenden Absatz vgl. Weiss, 1995, S. $87 \mathrm{ff}$.): Durch die gemeinsame Theorieentwicklung soll auf Basis der sehr unterschiedlichen handlungsleitenden Grundannahmen der Akteure ein gemeinsames Verständnis über eine übergeordnete Wirkungslogik hergestellt werden. Genau in dieser gemeinsamen Aushandlung und Verständigung liegt das Grundpotenzial des Ansatzes. Allerdings soll nicht darüber hinweggesehen werden, dass diese Konsensfindung sich schwierig und langwierig gestalten kann. Darüber hinaus ist es schwierig, unterschiedliche Schritte innerhalb der Wirkungslogik zu messen. Beispielsweise sind Haltungsänderungen oder das Verhalten in Kooperationsbeziehungen nur schwer über Indikatoren operationalisierbar. Daraus leitet sich auch die Frage ab, wie die zugrundeliegenden Wirkannahmen getestet werden und dabei alternati- 
ve Interpretationen von erhobenen Daten ausgeschlossen werden können. Dies erfordert sehr präzise Definitionen und Darstellungen der Wirkannahmen. Nicht zuletzt bleibt auch bei erfolgreich getesteten Wirkungslogiken die Frage, inwieweit sie generalisierbar und auf andere Kontexte übertragbar sind. Wirkungslogiken entstehen unter sehr spezifischen Umständen und werden konkret innerhalb der jeweiligen Akteurskonstellation erarbeitet. Es ist also zu beachten, dass ihre Evaluation keine allgemeingültigen Aussagen generieren kann - und diesen Anspruch auch gar nicht verfolgt.

Eine weitere Herausforderung im Kommunikationsprozess zur Erarbeitung einer Wirkungslogik besteht darin, dass sich die Akteure gegebenenfalls nur formal auf das gemeinsame Vorgehen im Rahmen der wirkungsorientierten Steuerung einigen, sich aber im alltäglichen Handeln nicht danach richten, sondern zweckrational nach eigenen Interessen vorgehen (Meyer \& Rowan, 1977). Ebenso ist fraglich, ob das geforderte iterative Vorgehen, also die regelmäßige Überarbeitung der Wirkungslogik auf Basis von Monitoringergebnissen, vor dem Hintergrund begrenzter zeitlicher Ressourcen realistisch ist.

Wirkungslogiken zwischen Bildungsverwaltung, Wissenschaft und pädagogischer Praxis gemeinsam $\mathrm{zu}$ erarbeiten ist also eine komplexe und ressourcenintensive Angelegenheit. Gleichzeitig scheint dies ein vielversprechender Ansatz zur Verbesserung der Kommunikation an den Schnittstellen zwischen Bildungsverwaltung, Bildungswissenschaft und Bildungspraxis zu sein.

\section{Literatur und Internetquellen}

Arbeitsgruppe „Lernen vor Ort” (2016). Einleitung: Zur Analyse von „Lernen vor Ort“. In Arbeitsgruppe "Lernen vor Ort" (Hrsg.), Kommunales Bildungsmanagement als sozialer Prozess. Studien $z u$ „Lernen vor Ort“ (S. 1-21). Wiesbaden: VS. https://doi. org/10.1007/978-3-658-12442-7_1

Astbury, B., \&. Leeuw, F. L. (2010). Unpacking Black Boxes: Mechanisms and Theory Building in Evaluation. American Journal of Evaluation, 31 (3), 363-381. https://doi. org/10.1177/1098214010371972

Böttcher, W. (2016). Absichten, Ziele und Wirkungen von Schulpolitik. Ein Aufruf, genauer über zielorientierte Steuerung nachzudenken. In I. Bormann, T. Brüsemeister \& S. Niedlich (Hrsg.), Transintentionalität im Bildungswesen (S. 55-71). Weinheim: Beltz Juventa.

Bronfenbrenner, U. (1979). The Ecology of Human Development. Experiments by Nature and Design. Cambridge, MA: Harvard University Press.

Chen, H. (1990). Theory-driven Evaluation. Thousand Oaks, CA: Sage.

Connell, J. P., \& Kubisch, A. C. (1998). Applying a Theory of Change Approach to the Evaluation of Comprehensive Community Initiatives: Progress, Prospects and Problems. In K. Fulbright-Anderson, A. C. Kubisch \& J. P. Connell (Hrsg.), New Approaches to Evaluating Community Initiatives, Vol. 2: Theory, Measurement and Analysis (S. 15-44). Queenstown, MD: Aspen Institute. 
Donaldson, S. I. (2007). Program Theory-driven Evaluation Science. Strategies and Applications. New York, NY: Taylor \& Francis. https://doi.org/10.4324/9780203809730

Funnell, S. C., \& Rogers, P. J. (2011). Purposeful Program Theory. Effective Use of Theories of Change and Logic Models. San Francisco, CA: Jossey-Bass.

Jungermann, A. (2018). Bildungslandschaften wirksam steuern. Eine heuristische Wirkungslogik unter Berücksichtigung des sozioökologischen Kontexts. Kumulative Dissertation an der TU Dortmund. Zugriff am 05.01.2021. Verfügbar unter: http://dx.doi.org/10.17877/ DE290R-19775.

Jungermann, A. (eingereicht). Shaping Supportive School-Community Ties. A Theory-led and Data-driven Framework for Analysis. Education and Urban Society.

Klawe, W. (2006). Multiperspektivische Evaluationsforschung als Prozess-Wirkungsrekonstruktion aus der Sicht der Beteiligten. In Deutsches Jugendinstitut e.V. (Hrsg.), Wirkungsevaluation in der Kinder- und Jugendhilfe. Einblicke in die Evaluationspraxis (S. 125-142). Augsburg: Pröll.

Manitius, V., \& Berkemeyer, N. (2011). Regionale Bildungsbüros - ein neuer Akteur der Schulentwicklung. In F. Dietrich, M. Heinrich \& N. Thieme (Hrsg.), Neue Steuerung alte Ungleichheiten? Steuerung und Entwicklung im Bildungssystem (S. 53-64). Münster: Waxmann.

Meyer, J., \& Rowan, B. (1977). Institutionalized Organizations: Formal Structure as Myth and Ceremony. American Journal of Sociology, 83 (2), 340-363. https://doi. org/10.1086/226550

Niedlich S., Lindner, M., \& Brüsemeister, T. (2014). Hierarchie und gleichberechtigte Kooperation im Schmelztiegel lokaler Governance - Zur „Contribution Analysis“ als einem möglichen Mittel zur Analyse kommunalen Bildungsmanagements. In K. Maag Merki, R. Langer \& H. Altrichter (Hrsg.), Educational Governance als Forschungsperspektive. Strategien. Methoden. Ansätze (2., erw. Aufl.) (S. 259-281). Wiesbaden: VS. https://doi.org/10.1007/978-3-658-06443-3_11

Patton, M. Q. (1986). Utilization-Focused Evaluation. Newbury Park, CA: Sage.

Patton, M. Q. (2011). Developmental Evaluation. Applying Complexity Concepts to Enhance Innovation and Use. New York, NY: The Guildford Press.

Schröder, J. (2001). Die unbekannte Dimension: Die Wirkung kommunaler Leistung. Vortrag auf der KGSt-Fachkonferenz „Controlling - zielgerichteter entscheiden und besser steuern" in Köln, 17. \& 18.05.2001.

Schröder, J., \& Kettiger, D. (2001). Wirkungsorientierte Steuerung in der sozialen Arbeit. Ergebnisse einer internationalen Recherche in den USA, den Niederlanden und der Schweiz (Schriftenreihe des Bundesministeriums für Familie, Senioren, Frauen und Jugend, Bd. 229). Stuttgart: BMFSFJ.

Weiss, C. H. (1995). Nothing as Practical as a Good Theory. Exploring Theory-based Evaluation for Comprehensive Community Initiatives for Children and Families. In J. I. Connell, A. C. Kubisch, L. B. Schorr \& C. H. Weiss (Hrsg.), New Approaches to Evaluating Community Initiatives: Concepts, Methods, and Contexts (S.65-92). Washington, DC: Aspen Institute.

Anja Jungermann, Dr., geb. 1984, Leiterin Regionales Bildungsbüro im Fachbereich Schule der Stadt Dortmund.

E-Mail: ajungermann@stadtdo.de

Korrespondenzadresse: Stadt Dortmund - Fachbereich Schule, Regionales Bildungsbüro, Königswall 25-27, 44137 Dortmund 